\title{
The Fundamental Neutron Physics Facilities at NIST
}

\author{
P. R. Huffman, M. Arif, M. S. Dewey, T. R. Gentile, \\ D. M. Gilliam, D. L. Jacobson, J. S. Nico, and A. K. Thompson
}

National Institute of Standards and Technology, Ionizing Radiation Division, Gaithersburg, MD 20899, USA

\begin{abstract}
The National Institute of Standards and Technology (NIST) Center for Neutron Research (NCNR) is a national center that provides thermal and cold neutrons beams for activities such as materials science, biological, polymer, and fundamental physics research. Four beams are currently in use for fundamental physics experiments. These include a neutron optics and interferometry facility, a $0.496 \mathrm{~nm}$ monochromatic beam, a $0.89 \mathrm{~nm}$ monochromatic beam, and a high-intensity polychromatic beam. Experiments primarily focus on studies of the weak interaction using neutrons. This paper provides a general overview of the facilities and highlights some current experiments.
\end{abstract}

The NIST Center for Neutron Research (NCNR) is located in Gaithersburg, MD and houses a $20 \mathrm{MW}$ research reactor that provides neutron beams for a wide variety of uses. These include materials science research, biological and polymer investigations, fundamental physics research, and neutron standards and dosimetry studies. Both thermal and cold neutron beams are available for use with instruments that include spectrometers, reflectometers, diffractometers, and small angle scattering chambers. Four beams are currently in use for fundamental physics experiments. These include three monochromatic neutron beams and a high-intensity polychromatic beam.

Neutrons are released in the reactor core from uranium fission products and are moderated to thermal energies by $\mathrm{D}_{2} \mathrm{O}$. Thermal beams are available for various neutron scattering instruments. One thermal beamport houses a large liquid hydrogen cold source. The cold source is a hollow shell with walls filled with liquid hydrogen at a temperature of $\approx 20 \mathrm{~K}$. Neutrons partially thermalize with the hydrogen, then exit towards a series of eight neutron guides that feed a set of cold neutron instruments. The cold neutrons pass into the open end of rectangular ${ }^{58} \mathrm{Ni}$-coated neutron guides $15 \mathrm{~cm}$ tall and $6 \mathrm{~cm}$ wide. ${ }^{58} \mathrm{Ni}$ has an effective neutron potential of $335 \mathrm{neV}$ such that neutrons with low enough momentum perpendicular to the surface will be totally reflected and thus can be transported to the far end of the guides (that are typically $20 \mathrm{~m}$ to $60 \mathrm{~m}$ in length) with minimal losses. For a fixed neutron wavelength (or energy), the surfaces of the guide are reflecting up to a critical angle of $\approx 20 \mathrm{mrad} \mathrm{nm}^{-1}$.

This paper will provide a brief description of the four cold neutron beams that are available for fundamental

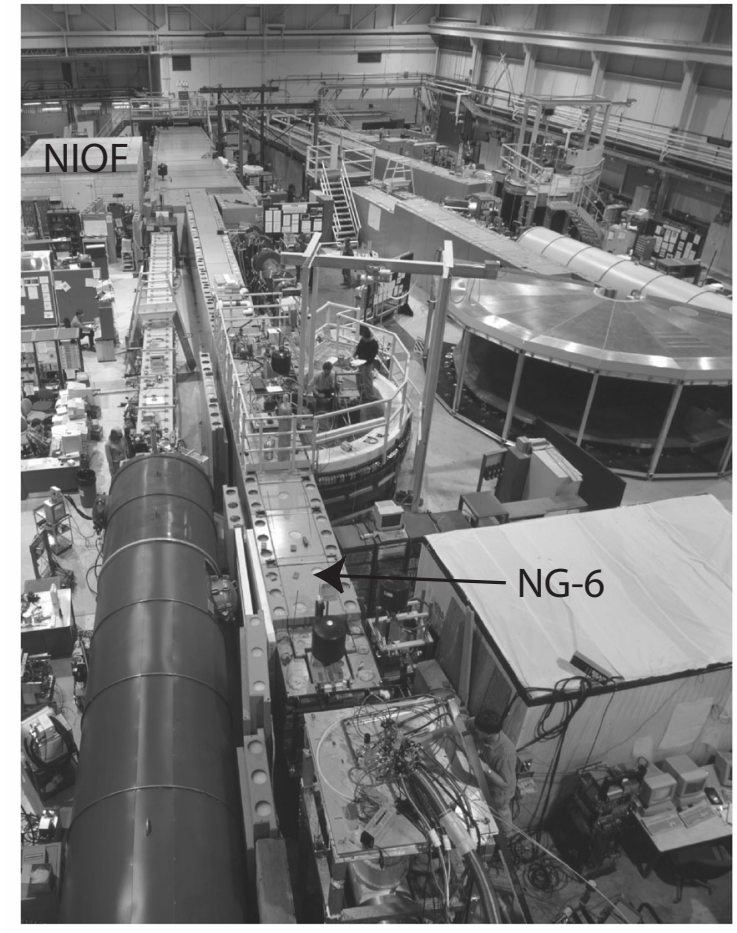

FIGURE 1. Photograph of the neutron guide hall at the NCNR. This paper describes the NIOF and NG-6 neutron facilities. Three monochromatic beams and one polychromatic beam is available for use in fundamental physics research.

neutron physics research. Research includes investigations of the weak interaction and tests of the Standard Model through the study of neutron decay and neutron interactions. Highlights of some recent experiments per- 


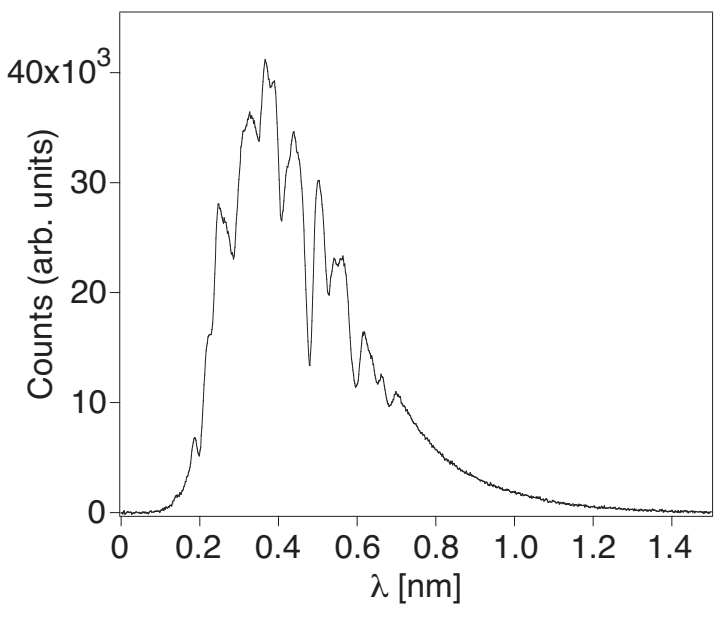

FIGURE 2. Spectrum of the filtered neutron wavelengths in the NG-6 polychromatic beam. The dips in the spectrum correspond to Bragg-edges from materials upstream, with the largest at $0.48 \mathrm{~nm}$ arising from an upstream monochromator. The data were taken with a $15 \mathrm{~cm}$ thick bismuth filter.

formed at the NCNR fundamental physics facilities will be presented.

\section{BEAMLINES}

Three of the fundamental physics beamlines reside at the end of cold neutron guide number six (NG-6): a polychromatic beam, a $0.89 \mathrm{~nm}$ monochromatic beam, and a $0.496 \mathrm{~nm}$ monochromatic beam. The fourth resides on neutron guide number seven (NG-7) and is directed into the NIOF.

\section{Polychromatic Beam}

Neutrons exiting from the upper $7 \mathrm{~cm}$ of the NG-6 guide pass through a ${ }^{6} \mathrm{Li}$-loaded-glass collimator that defines a $6 \mathrm{~cm}$ diameter beam. The wavelength distribution of the beam is roughly Maxwellian with an average temperature of $30 \mathrm{~K}$. To minimize backgrounds for neighboring experiments, single crystal, cooled $(77 \mathrm{~K})$ bismuth filters (5 $\mathrm{cm}$ to $15 \mathrm{~cm}$ long) are placed in the beam to attenuate gamma rays from upstream components in the guide. The neutron fluence rate with $15 \mathrm{~cm}$ of bismuth and no additional filters is $(1.5 \pm 0.1) \times 10^{9} \mathrm{~cm}^{-2} \mathrm{~s}^{-1}$. The maximum fluence rate (ie. no filter) is approximately $2.3 \times 10^{9} \mathrm{~cm}^{-2} \mathrm{~s}^{-1}$. A wavelength spectrum of the neutron beam is shown in Fig. 2 with $15 \mathrm{~cm}$ of single crystal bismuth filters in place.

The thermal energy (short wavelength) neutrons can be filtered from the beam using Bragg scattering from polycrystalline materials; the most common being $5 \mathrm{~cm}$ to $15 \mathrm{~cm}$ thick of beryllium. Beryllium will Bragg reflect neutrons out of the beam whose wavelengths are less than $0.395 \mathrm{~nm}$. Other filter materials that have been used are graphite and polycrystalline bismuth.

Experiments requiring unpolarized neutrons can use the exiting beam directly, while other experiments require the neutrons to be polarized. Two polarization technologies are available: supermirror and ${ }^{3} \mathrm{He}$ transmission polarizers. Polarizations of $96 \%$ are typical for the supermirror device, with the polarized neutron beam slightly more divergent and reflected by an angle of approximately $1^{\circ}$ with respect of the primary beam. ${ }^{3} \mathrm{He}$ transmission polarizers are generally superior for highly accurate neutron polarization measurements, polarization of large diameter beams, and polarization over a wavelength spectrum larger than can be accomodated by supermirrors.

\subsection{6 nm Monochromatic Beam}

Two monochromators are positioned in the lower $8 \mathrm{~cm}$ of the polychromatic beam exiting the NG-6 guide. The upstream monochromator consists of a piece of pyrolytic graphic $5.1 \mathrm{~cm}$ by $5.1 \mathrm{~cm}$ by $0.2 \mathrm{~cm}$ thick with a mosaic of $0.8^{\circ}$. This crystal Bragg reflects a $0.496 \mathrm{~nm}$ beam at an angle of approximately $104^{\circ}$ with respect to the primary beam.

Once the neutron beam reflects from the monochromator, it passes through $10 \mathrm{~cm}$ of cooled beryllium to remove the $\lambda / 2$ component of the beam. The neutron flence rate available at a typical experimental position (approximately $2 \mathrm{~m}$ from the crystal) is $6.5 \times$ $10^{5} \mathrm{~cm}^{-2} \mathrm{~s}^{-1}$.

The $0.496 \mathrm{~nm}$ neutron beam is used for neutron calorimetry experiments, polarized ${ }^{3} \mathrm{He}$ development, and for neutron dosimetry work.

\subsection{9 nm Monochromatic Beam}

Downstream of the $0.496 \mathrm{~nm}$ monochromator resides a $0.89 \mathrm{~nm}$ stage 2 potassium-intercalated graphite monochromator. The outgoing $0.89 \mathrm{~nm}$ neutron beam is $6 \mathrm{~cm}$ by $6 \mathrm{~cm}$ and is directed at an angle of $60^{\circ}$ with respect to the polychromatic beam.

The individual monochromator pieces have high stage purity and mosaics in the range of $1.1^{\circ}$ to $2.1^{\circ}$. The reflectivity between $0.88 \mathrm{~nm}$ and $0.90 \mathrm{~nm}$ is $(85.4 \pm$ $0.5) \%$, yielding a capture fluence rate at $1 \mathrm{~m}$ from the crystal of $4.7 \times 10^{6} \mathrm{~cm}^{-2} \mathrm{~s}^{-1}$.

Two additional wavelengths are present, one at $\lambda / 2=$ $0.45 \mathrm{~nm}$ and one at $\lambda / 3=0.30 \mathrm{~nm}$. The $\lambda / 2$ peak is re- 


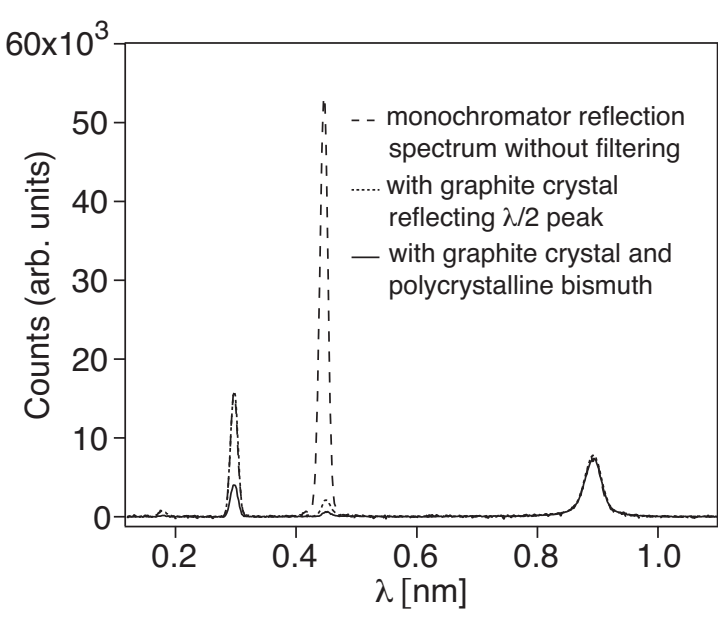

FIGURE 3. The wavelength spectrum of the $0.89 \mathrm{~nm}$ beam before and after filtering.

moved by a pyrolytic graphite crystal with a $10^{\circ}$ mosaic positioned to reflect out the $0.45 \mathrm{~nm}$ neutrons. The $\lambda / 3$ peak is minimized using Bragg reflection from polycrystalline bismuth. A time-of-flight spectrum of the $0.89 \mathrm{~nm}$ neutron beam before and after wavelength filtering is shown in Fig. 3. The $0.89 \mathrm{~nm}$ beam is used primarily for ultracold neutron production.

\section{Neutron Interferometric and Optics Facility}

The NIOF is one of the world's premier user facilities for neutron interferometry and related neutron optical measurements. A neutron interferometer (NI) splits, then recombines neutron waves. This gives the NI its unique ability to experimentally access the phase of neutron waves. Phase measurements are used to study the magnetic, nuclear, and structural properties of materials, as well fundamental questions in quantum physics. An overview of the facility can be found in Ref. [1].

Neutrons are extracted from the primary NG-7 beam using a dual-crystal parallel-tracking pyrolytic graphite monochromator system. Monochromatic beams (either unpolarized or polarized) with wavelengths from $0.2 \mathrm{~nm}$ to $0.48 \mathrm{~nm}$ are available. Neutrons are counted with integrating ${ }^{3} \mathrm{He}$ detectors or by high resolution positionsensitive detectors (with a resolution better than $50 \mu \mathrm{m}$ ). The sensitivity of the apparatus is greatly enhanced by state-of-the-art thermal, acoustical and vibration isolation systems. To reduce vibration, the NIOF is built on its own foundation, separate from the rest of the building. The result is a NI facility with exceptional phase stability $(0.25 \%$ day) and high contrast $(90 \%)$. The beam at the interferometer is $2 \mathrm{~mm}$ wide by $8 \mathrm{~mm}$ tall and has a fluence rate of $2 \times 10^{5} \mathrm{~cm}^{-2} \mathrm{~s}^{-1}$. A picture of the facility is

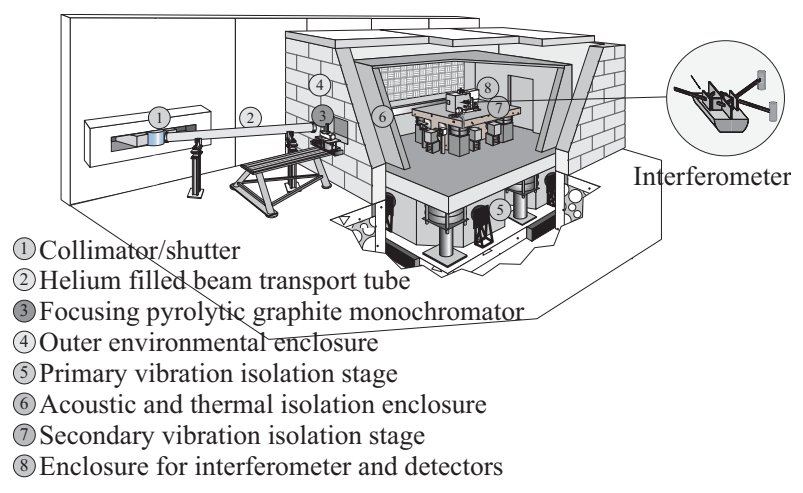

FIGURE 4. Neutron Interferometer and Optics Facility

shown in Fig. 4.

\section{EXPERIMENTS}

\section{Neutron Lifetime Measurements}

Two experiments are underway to measure the lifetime of the free neutron. The first uses a Penning-trap technique and is nearing completion. The second technique is under development and involves three-dimensional magnetic confinement of neutrons.

In the Penning-trap technique, when a neutron in the beam decays while passing through the trapping region, the recoil proton is trapped. The proton is held in a $5 \mathrm{~T}$ magnetic field that provides radial containment and an electrode at each end to provide axial confinement. Periodically, one of the end electrodes is lowered to ground potential to allow the accumulated trapped proton(s) to exit the trap and be counted by a proton detector. By knowing precisely the number of neutrons that decay while in the trapping volume and the number that pass through, the neutron lifetime can be extracted. Additional details are found in Ref. [2].

Determination of the lifetime requires either an accurately known trap length or a variable trap length with accurately known differences in length. The latter is more easily realized physically and used in the current design. The required accuracy for determination of neutron flux (or density) is the primary systematic limitation. The neutron monitor employed is known as a $1 / v$ detector, with an efficiency that is weighted by the reciprocal of the neutron velocity. This ${ }^{6} \mathrm{Li}$ detector is a transmission device that is nearly transparent to neutrons. By measuring rate of alpha production from this well-characterized foil, the neutron flux can be determined.

The preliminary lifetime value obtained in this experiment is $\tau_{n}=(884.6 \pm 4.1) \mathrm{s}$ [3]. Continuing efforts to measure the neutron count rate are underway by calori- 
metric techniques, that should reduce the final uncertainty by a factor of two.

The second experiment under development for measuring the neutron lifetime uses magnetic trapping techniques. In brief, ultracold neutrons (UCN) are produced by inelastic scattering of cold $(0.89 \mathrm{~nm})$ neutrons in a reservoir of superfluid ${ }^{4} \mathrm{He}$ (the "superthermal" process) [4]. These neutrons are then confined by a threedimensional magnetic trap. As the trapped neutrons beta decay, the energetic electrons produced generate scintillations in the liquid $\mathrm{He}$ [5], that should be detectable with nearly $100 \%$ efficiency. $\tau_{n}$ can be directly determined by measuring the scintillation rate as a function of time. A proof-of-principle demonstration of this technique has been performed [6]. The apparatus has been upgraded and is now equipped with a larger magnet that will be used to make a measurement of the neutron lifetime at the $10^{-3}$ level.

The Penning-trap measurements were performed on the polychromatic beam, while the UCN lifetime work is underway on the $0.89 \mathrm{~nm}$ monochromatic beam.

\section{Time-Reversal Violation}

The EMIT experiment searches for the time-reversal violating $D$-coefficient in neutron beta decay. A description of the experiment can be found in Ref. [7].

The detection region consists of four $50 \mathrm{~cm}$ long electron detectors and four $30 \mathrm{~cm}$ long proton detectors arranged octagonally around the polarized neutron beam. The decay electrons and protons are detected in coincidence for neutrons polarized perpendicular to the direction of the neutron momentum. A difference in the coincidence rate for neutrons in one spin direction versus the opposite spin direction is a direct indication of timereversal violation.

Data was taken in initial runs and set a limit of $D=[-0.6 \pm 1.2($ stat $) \pm 0.5($ syst $)] \times 10^{-3}$. Substantial improvements have been made with a number of components including the proton detectors and the experiment is presently taking data on the polychromatic beam. These improvements are expected to produce a factor of ten higher data rate with significantly reduced systematic effects. It should be feasible to reduce the statistical uncertainty on $D$ to $2 \times 10^{-4}$.

\section{Polarized ${ }^{3}$ He program}

Polarized neutrons are preferred or required for many of the experiments underway. The ${ }^{3} \mathrm{He}$ spin filter, that operates by the absorption of one spin state, does not affect the other spin state so no deflection or divergence is introduced into the beam. Because of the simple $1 / v$ ${ }^{3} \mathrm{He}$-neutron cross section, it can serve well as a broadband neutron polarizer.

We are developing practical neutron spin filters based on two different techniques for polarizing ${ }^{3} \mathrm{He}$ : metastability-exchange optical pumping and spinexchange optical pumping. An overview of the two polarization techniques and a description of the neutron ${ }^{3} \mathrm{He}$ program at NIST can be found in Ref. [8].

Polarized ${ }^{3} \mathrm{He}$ spin filters are based on the spin dependence of the ${ }^{3} \mathrm{He}$-neutron capture cross section. Because the two neutrons in the ${ }^{4} \mathrm{He}$ nucleus are spin paired, only neutrons of one spin state are absorbed by the ${ }^{3} \mathrm{He}$ nucleus to form a ${ }^{4} \mathrm{He}$ nucleus. For the ${ }^{3} \mathrm{He}$ spin parallel to the neutron spin, the thermal capture cross section is essentially zero, whereas for the spins anti-parallel, the cross section is $10666 \mathrm{~b}$.

Using both techniques, we have demonstrated $50 \%$ to $60 \%{ }^{3} \mathrm{He}$ polarization under the conditions that are relevant to neutron polarizers. For a ${ }^{3} \mathrm{He}$ polarization of $60 \%$, the neutron polarization is $90 \%$ and the transmission is $18 \%$. We are also able to analyze the neutron polarization using ${ }^{3} \mathrm{He}$-based techniques.

\section{SUMMARY}

We operate one end-guide position that is wholly dedicated to fundamental neutron physics research. This station presently consists of a primary high-flux polychromatic beam, a $0.49 \mathrm{~nm}$ monochromatic beam, and a $0.89 \mathrm{~nm}$ monochromatic beam for ultracold neutron production. In addition, we have a state-of-the-art neutron interferometry and optics facility.

The neutron beams are available to users for experiments that fall in line with the general missions of the facility.

\section{REFERENCES}

1. Arif, M., et al., in Proceedings of the SPIE - The International Society for Optical Engineering, edited by C. G. Gordon, Proc. SPIE 2264, 1994, pp. 20-26.

2. Snow, W. M., et al., Nucl. Instrum. Meth. A 440, 528-534 (2000).

3. Dewey, M. S., et al., NIST Special Publication 977, 6 (2002).

4. Golub, R. and Pendlebury, J. M., Rep. Progr. Phys. 42, 439-501 (1979).

5. Stockton, M., Keto, J. W., and Fitzsimmons, W. A., Phys. Rev. Lett. 24, 654-657 (1970).

6. Huffman, P. R., et al., Nature 403, 62-64 (2001).

7. Lising, L. J., et al., Phys. Rev. C 62, 055501 (2000).

8. Rich, D. R., et al., Physica B 305, 203-207 (2001). 